\title{
Kinematics and dynamics of the M 51-type galaxy pair NGC 3893/96 (KPG 302)
}

\author{
I. Fuentes-Carrera ${ }^{1, \star}$, M. Rosado ${ }^{2}$, P. Amram ${ }^{3}$, H. Salo ${ }^{4}$, and E. Laurikainen ${ }^{4}$
}

\author{
1 Instituto de Astronomía, Geofísica e Ciencias Atmosféricas, Universidade de São Paulo, Rua do Matão 1226-Cidade Universitária, \\ 05508-900 São Paulo SP, Brazil \\ e-mail: isaura@astro.iag.usp.br, isaura.fuentes@obspm.fr \\ 2 Instituto de Astronomía, Universidad Nacional Autónoma de México (UNAM), Apdo. Postal 70-264, 04510, México, D.F., México \\ e-mail: margarit@astroscu. unam.mx \\ ${ }_{3}$ Laboratoire d'Astrophysique de Marseille, 2 place Le Verrier, Marseille Cedex 4, France \\ e-mail: philippe.amram@oamp.fr \\ 4 Department of Physical Sciences, Division of Astronomy, University of Oulu, 90570 Oulu, Finland \\ e-mail: heikki.salo@oulu.fi,eija.laurikainen@oulu.fi
}

Received 9 January 2007 / Accepted 23 January 2007

\section{ABSTRACT}

\begin{abstract}
Aims. We study the kinematics and dynamics of the M 51-type interacting galaxy pair KPG 302 (NGC 3893/96). We analyze the perturbations induced by the encounter on each member of the pair, as well as the distribution of the dark matter (DM) halo of the main galaxy in order to explore possible differences between DM halos of "isolated" galaxies and those of galaxies belonging to a pair.

Methods. The velocity field of each galaxy was obtained using scanning Fabry-Perot interferometry. A two-dimensional kinematic and dynamical analysis of each galaxy and the pair as a whole are done emphasizing the contribution of circular and non-circular velocities. Non-circular motions can be traced on the rotation curves of each galaxy allowing us to differentiate between motions associated to particular features and motions that reflect the global mass distribution of the galaxy. For the main galaxy of the pair, NGC 3893, optical kinematic information is complemented with HI observations from the literature to build a multi-wavelength rotation curve. We try to fit this curve with a mass-distribution model using different DM halos.

Results. Non-circular motions are detected on the velocity fields of both galaxies. These motions can be associated to perturbations due to the encounter and, in the case of the main galaxy, to the presence of a structure such as spiral arms. The location of the corotation radius of this galaxy is also explored. We find that the multi-wavelength rotation curve of NGC 3893, "cleaned" from the effect of non-circular motions, cannot be fitted whether by a pseudo-isothermal or by a NFW DM halo.
\end{abstract}

Key words. galaxies: interactions - galaxies: kinematics and dynamics - galaxies: individual: NGC 3893, NGC 3896 galaxies: spiral - galaxies: halos

\section{Introduction}

The difference between the mass distribution implied by the luminosity of a disk galaxy and the distribution of mass implied by the rotation velocities offers strong evidence that disk galaxies are embedded in extended halos of dark matter (Sofue \& Rubin 2001, and references therein). Detailed knowledge of dark matter (DM) halos around galaxies holds important clues to the physics of galaxy formation and evolution and is an essential ingredient for any model aiming to link the observable Universe with cosmological theories. In practice, realistic DM halos are neither static nor spherically symmetric (Knebe et al. 2004) and it is still unknown if their structure and distribution is intrinsically related to the environment of their galaxies. The question remains as to whether an intrinsic difference exists between the DM halo of an "isolated" galaxy, the DM halo of a galaxy belonging to a pair or that of a galaxy that is part of a larger group such as a compact group or a cluster.

In this sense, rotation curves (RCs) are a powerful tool for studying the distribution of matter (both baryonic and nonbaryonic) in interacting disk galaxies. For a description of

\footnotetext{
* Presently at the Observatoire de Paris-Meudon.
}

the classical method for studying mass distribution, see BlaisOuellette et al. (2001), and references therein. The RCs also allow us to determine the maximum rotation velocity of a galaxy and thus infer the total mass within a certain radius using methods such as that of Lequeux (1983). Nevertheless, care must be taken when using kinematic information from interacting galaxies, since they are subject to kinematical perturbations that may affect the correct determination of an RC that actually traces the global mass distribution of the galaxy. For this reason, 3D spectroscopy observations are required to separate circular from noncircular motions in the velocity field of a galaxy and its rotation curve as shown in Fuentes-Carrera et al. (2004).

In this work, we present scanning Fabry-Perot observations of the M 51-type interacting galaxy pair NGC 3893/96 (KPG 302). Section 2 presents the scanning Fabry-Perot (FP) observations and data reductions. Section 3 introduces the pair of galaxies KPG 302 (NGC 3893/96). In Sect. 4 we present the kinematic information derived from the F-P observations. Section 5 presents the dynamical analysis of both galaxies, mass estimates and the mass distribution for NGC 3893. The discussion and conclusions are presented in Sects. 6 and 7, respectively. 


\section{Observations and data reductions}

Observations of NGC 3893/96 (KPG 302) were done at the $2.1 \mathrm{~m}$ telescope at the OAN-SPM (México) using the scanning Fabry-Perot interferometer PUMA (Rosado et al. 1995). PUMA is a focal reducer built at the Instituto de Astronomía-UNAM used to make direct images and Fabry-Perot (FP) interferometry of extended emission sources (field of view 10'). The FP used is an ET-50 (Queensgate Instruments) with a servostabilization system having a free spectral range of $19.95 \AA\left(912 \mathrm{~km} \mathrm{~s}^{-1}\right)$ at $\mathrm{H} \alpha$. Its finesse $(\sim 24)$ leads to a sampling spectral resolution of $0.41 \AA\left(19.0 \mathrm{~km} \mathrm{~s}^{-1}\right)$ achieved by scanning the interferometer free spectral range through 48 different channels. A $1024 \times 1024$ Tektronix CCD detector with a resolution of $0.58^{\prime \prime} /$ pixel was used. We used a $2 \times 2$ binning to enhance the signal. The final spatial sampling equals $1.16^{\prime \prime} /$ pixel. To isolate the redshifted $\mathrm{H} \alpha\left(\lambda_{\text {at rest }}=6562.73 \AA\right)$ emission of the galaxies, we used an interference filter centered at $6584 \AA$ with an $F W H M$ of $10 \AA$. To average the sky variations during the exposure, we got two data cubes with an exposure time of 48 min each $(60 \mathrm{~s}$ per channel). These data cubes were co-added leading to a total exposure time of 96 minutes. For the calibration, we used a $\mathrm{H}$ lamp whose $6562.78 \AA$ line was close to the redshifted nebular wavelength. Two calibration cubes were obtained at the beginning and at the end of the galaxy observation to check the metrology.

Data reduction and analysis were done using mainly the $\mathrm{ADHOCw}^{1}$ and CIGALE softwares (LeCoarer et al. 1993). Standard corrections (cosmic rays removal, bias subtraction, flat-fielding, etc.) were done on each cube. Once the object cubes were co-added, the night sky continuum and $6577.3 \AA$ $\mathrm{OH}$ sky line were subtracted. A spectral Gaussian smoothing $\left(\sigma=57 \mathrm{~km} \mathrm{~s}^{-1}\right)$ was also performed. Once the spectral smoothing was done, the calibration in wavelength was fixed for each profile at each pixel using the calibration cube. The Fabry-Perot scanning process allows us to obtain a flux value at pixel level for each of the 48 scanning steps. The intensity profile found along the scanning process contains information about the monochromatic emission $(\mathrm{H} \alpha)$ and the continuum emission of the object. The continuum image computation was done considering the mean of the 3 lowest intensities of the 48 channels cube. For the monochromatic image, the $\mathrm{H} \alpha$ line intensity was obtained by integrating the monochromatic profile in each pixel. The velocity maps were computed using the barycenter of the $\mathrm{H} \alpha$ profile peaks at each pixel. To get a sufficient signal-to-noise ratio on the outer parts of each galaxy, we performed three spatial Gaussian smoothings $\left(\sigma=2.36,3.54,4.72^{\prime \prime}\right)$ on the resulting calibrated cube. A variable-resolution radial velocity map was built using high spatial resolution (less spatially-smoothed pixels) for regions with an originally higher signal-to-noise ratio.

\section{NGC 3893 and NGC 3896}

NGC 3893/96 is an interacting galaxy pair with number 302 in the Catalogue of Isolated Pairs of Galaxies in the Northern Hemisphere (KPG, Karachentsev 1972). Morphologically, it resembles M 51 (NGC 5194/95) since it is composed of a main spiral galaxy and a considerably smaller companion. KPG 302 is situated in the Ursa Major cluster. With only 79 members, this cluster is poorly defined with a velocity dispersion of only $148 \mathrm{~km} \mathrm{~s}^{-1}$ and a virial radius of $880 \mathrm{kpc}$ (Tully et al. 1996). It essentially contains only late-type galaxies distributed with no

\footnotetext{
1 http://www.oamp.fr/adhoc/adhocw.htm developed by J. Boulesteix.
}

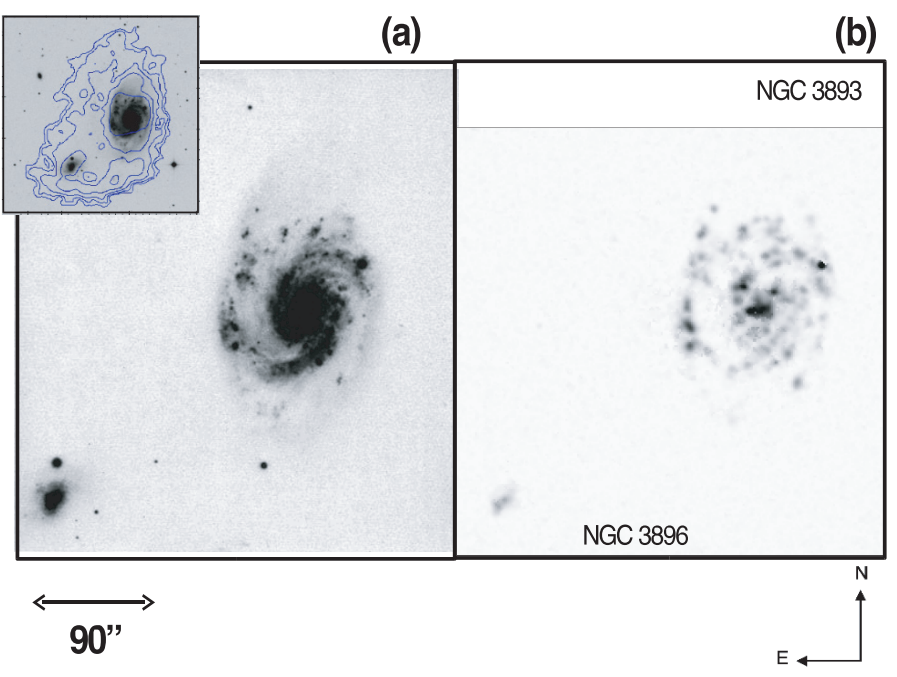

Fig. 1. a) Direct B image of NGC 3893/96 (KPG 302) from "The Carnegie Atlas of Galaxies. Volume II" (Sandage \& Bedke 1994). b) Monochromatic $H \alpha$ (continuum subtracted) image of the pair obtained from the scanning Fabry-Perot interferometer PUMA data cubes. Upper panel: Optical image with HI isophotes superposed. Image taken from Verheijen \& Sancisi (2001) in "An HI Rogues Gallery" (http://www.nrao.edu/astrores/HIrogues/webGallery/ RoguesGallery06.html)

particular concentration toward the center. Given the isolation criteria used in the KPG and the nature of the cluster, it is possible that the DM halo (or halos) of the galaxies in the pair are isolated from those of the cluster.

NGC 3893 is a grand-design spiral similar to NGC 5194 in M 51 (Fig. 1a). It has been classified as SABc in LEDA database, and as $\mathrm{SAB}(\mathrm{rs}) \mathrm{c}$ in the $\mathrm{NED}^{2}$ database and in the RC3 (de Vaucouleurs et al. 1991). However, Hernández-Toledo \& Puerari (2001) classify it as a non-barred galaxy without any inner ring. The HI observations (Verheijen \& Sancisi 2001) show that this galaxy is slightly warped in its outer parts - both in its HI distribution and its HI kinematics. Its companion, NGC 3896, appears to be an intermediate type galaxy between and S0 and a spiral, which also has a bar. It shows extended $\mathrm{H} \alpha$ emission. NGC 3893 shows no color excess, whereas NGC 3896 has a predominantly blue $B-V$ color in the central parts of the galaxy (Laurikainen et al. 1998; Hernández-Toledo \& Puerari 2001).

The star-formation rate (SFR) of each member of the pair was derived by James et al. (2004). These authors find a value of $5.62 M_{\odot} \mathrm{yr}^{-1}$ for NGC 3893 and a value of $0.14 M_{\odot} \mathrm{yr}^{-1}$ for its companion. NGC 3893 was also part of a dynamical analysis of high surface brightness spiral galaxies using long-slit observations and numerical modeling in order to quantify the luminousto-dark matter ratio inside their optical radii (Kranz et al. 2003). According to these authors, NGC 3893 has a massive stellar disk that dominates the dynamics of the central regions with a disk mass of $2.32 \times 10^{10}$ solar masses. They infer the location of the corotation resonance of this galaxy at $5.5 \pm 0.5 \mathrm{kpc}$. Though optical images of this pair do not show an apparent connection between the two galaxies, radio images by Verheijen \& Sancisi (2001) show extended HI emission encompassing both galaxies (small panel in Fig. 1). This common HI envelope is elongated from SE to NW, parallel to the line that joins the nuclei of both galaxies. HI isophotes also show what could be considered as a

2 The NASA/IPAC Extragalactic Database (NED) is operated by the Jet Propulsion Laboratory, California Institute of Technology, under contract with the National Aeronautics and Space Administration. 
Table 1. Parameters of NGC 3893 and NGC 3896.

\begin{tabular}{|c|c|c|}
\hline & NGC 3893 & NGC 3896 \\
\hline Coordinates $(\mathrm{J} 2000)^{a}$ & $\begin{array}{c}\alpha=11 \mathrm{~h} 48 \mathrm{~m} 38.38 \\
\delta=+48^{\circ} 42^{\prime} 34.4^{\prime \prime}\end{array}$ & $\begin{array}{c}\alpha=11 \mathrm{~h} 48 \mathrm{~m} \mathrm{56.42 \textrm {s }} \\
\delta=+48^{\circ} 40^{\prime} 29.2^{\prime \prime}\end{array}$ \\
\hline \multirow[t]{3}{*}{ Morphological type } & $\mathrm{SABc}^{a}$ & $\mathrm{~S} 0-\mathrm{a}^{a}$ \\
\hline & $\mathrm{SAB}(\mathrm{rs}) \mathrm{c}^{b, c}$ & $\mathrm{~S} 0 / \mathrm{a}^{c}: \mathrm{pec}^{b}$ \\
\hline & $\mathrm{Sc}^{d}$ & $\mathrm{SBbc} \mathrm{pec}^{d}$ \\
\hline$m_{B}^{d}(\mathrm{mag})$ & 11.13 & 14.05 \\
\hline$B-V^{d}$ (in mag) & 0.56 & 0.46 \\
\hline$D_{25} / 2^{a}\left({ }^{\prime}\right)$ & 2.03 & 0.75 \\
\hline Distance $^{e}(\mathrm{Mpc})$ & 18.6 & 18.6 \\
\hline $\operatorname{SFR}^{f}\left(M_{\odot} / \mathrm{yr}\right)$ & 5.62 & 0.14 \\
\hline \multirow[t]{4}{*}{ Heliocentric systemic velocity $\left(\mathrm{km} \mathrm{s}^{-1}\right)$} & $969 \pm 3^{a, g}$ & $959 \pm 12^{a, g}$ \\
\hline & $973^{j}$ & - \\
\hline & $962.0 \pm 5^{h}$ & $920 \pm 5^{h}$ \\
\hline & $958.0^{k}$ & - \\
\hline \multirow[t]{5}{*}{$V_{\text {rotmax }}\left(\mathrm{km} \mathrm{s}^{-1}\right)$} & $251.2^{a, g}$ & $150^{a, g}$ \\
\hline & $195^{j}$ & confused ${ }^{j}$ \\
\hline & $220 \pm 3^{l}$ & - \\
\hline & $197 \pm 10^{h}$ & $50 \pm 10^{h}$ \\
\hline & $207^{k}$ & $-\ldots$ \\
\hline \multirow[t]{4}{*}{$\mathrm{PA}\left({ }^{\circ}\right)$} & $352^{j}$ & $-\ldots$ \\
\hline & $166+180^{m}$ & $-\ldots$ \\
\hline & $340 \pm 10^{h}$ & $294 \pm 5^{h}$ \\
\hline & $167+180^{\mathrm{k}}$ & $-\ldots$ \\
\hline \multirow[t]{4}{*}{ Inclination $\left({ }^{\circ}\right)$} & $49 \pm 2^{i}$ & $48 \pm 3^{i}$ \\
\hline & $42^{m}$ & - - \\
\hline & $45 \pm 3^{h}$ & $49 \pm 3^{h}$ \\
\hline & $49^{k}$ & $-\ldots$ \\
\hline$m_{K}^{n}$ (mag) & 7.891 & 11.648 \\
\hline
\end{tabular}

${ }^{a}$ LEDA database. ${ }^{b}$ NED database. ${ }^{c}$ RC3 (de Vaucouleurs et al. (1991). ${ }^{d}$ Hernández-Toledo \& Puerari (2001). ${ }^{e}$ Tully \& Pierce (2000). ${ }^{f}$ James et al. (2004), total measured $\mathrm{H} \alpha+[\mathrm{NII}]$ line flux corrected for [NII] contamination. ${ }^{g}$ From HI observations. ${ }^{h}$ This work. ${ }^{i}$ Verheijen (2001), HI observations. ${ }^{j}$ Verheijen \& Sancisi (2001), HI observations. ${ }^{k}$ Garrido et al. (2002). ${ }^{l}$ Tully et al. (1996). ${ }^{m}$ Kranz, Slyz \& Rix (2003). ${ }^{n}$ 2MASS (Skrutskie et al. 2006) $K_{\text {ext }}$ value taken from the NED database.

broad arm going from NGC 3893 to NGC 3896. Table 1 lists the main parameters of each galaxy.

\section{Kinematic results}

\subsection{Monochromatic images}

Figure $1 \mathrm{~b}$ displays the monochromatic $\mathrm{H} \alpha$ image of the pair. Knotty HII regions lie along the spiral arms of NGC 3893. Although they mainly follow the two main arms, they also outline small segments of fainter and less pronounced flocculent arms. Intense HII regions are seen on the east side of the galaxy along the western spiral arm. A very bright HII region is seen in the central parts of the galaxy.

For NGC 3896, significant $\mathrm{H} \alpha$ emission is seen within the inner 13.9" $(1.3 \mathrm{kpc})$ southeast of the center of the galaxy. This emission displays two maxima that form a rather elongated region (left panel in Fig. 1b). The northern parts of the galaxy show weak diffuse emission. This type of emission is also seen on the western side of the galaxy. These sides are closer to NGC 3893.

\subsection{Velocity fields}

The upper panel in Fig. 2 shows the velocity field of NGC 3893. It is a smooth and regular field; the isovelocities show no significant distortions. Although the galaxy has an elongated inner structure in the monochromatic image (see Fig. 1), the central parts of the galaxy show no kinematic signatures that could be associated to the presence of a nuclear bar. The minor kinematic axis is almost perpendicular to the main axis of the galaxy, outlined by isovelocities with $955 \mathrm{~km} \mathrm{~s}^{-1}$. Small wiggles seen along these isovelocities could be a signature of an inner ring, similar to what is shown by the simulations by Salo et al. (1999) of IC4214. The velocity field is symmetrical with respect to the kinematical minor axis. Locally, minor irregularities are seen in the distribution of radial velocities, especially along the spiral arms of the galaxy. These might be associated to the passage of gas through the spiral density wave. For NGC 3896, the velocity field is very perturbed, displaying a mild velocity gradient from the SE to the NW (upper panel in Fig. 3). Isovelocities are very patchy and crooked.

\subsection{Rotation curves}

In the case of early-stage interactions, the inner parts of galaxies are not strongly perturbed, velocity fields are still smooth and symmetrical, resulting in symmetric and low-scattered rotation curves (RCs) up to a certain radius (Fuentes-Carrera et al. 2004). With this assumption in mind, the RC of each galaxy was computed considering different values for the kinematical parameters involved in order to obtain a symmetric curve in the inner parts of the galaxy and to minimize scatter on each side of the curve. The rotation curves are sampled with bins of 2 pixels $\left(\sim 2.3^{\prime \prime}\right)$. Error bars give the dispersion of the rotation velocities computed for all the pixels found inside each elliptical ring defined by the successive bins. This approach is described in more detail in Fuentes-Carrera et al. (2004) and Garrido et al. (2005). 

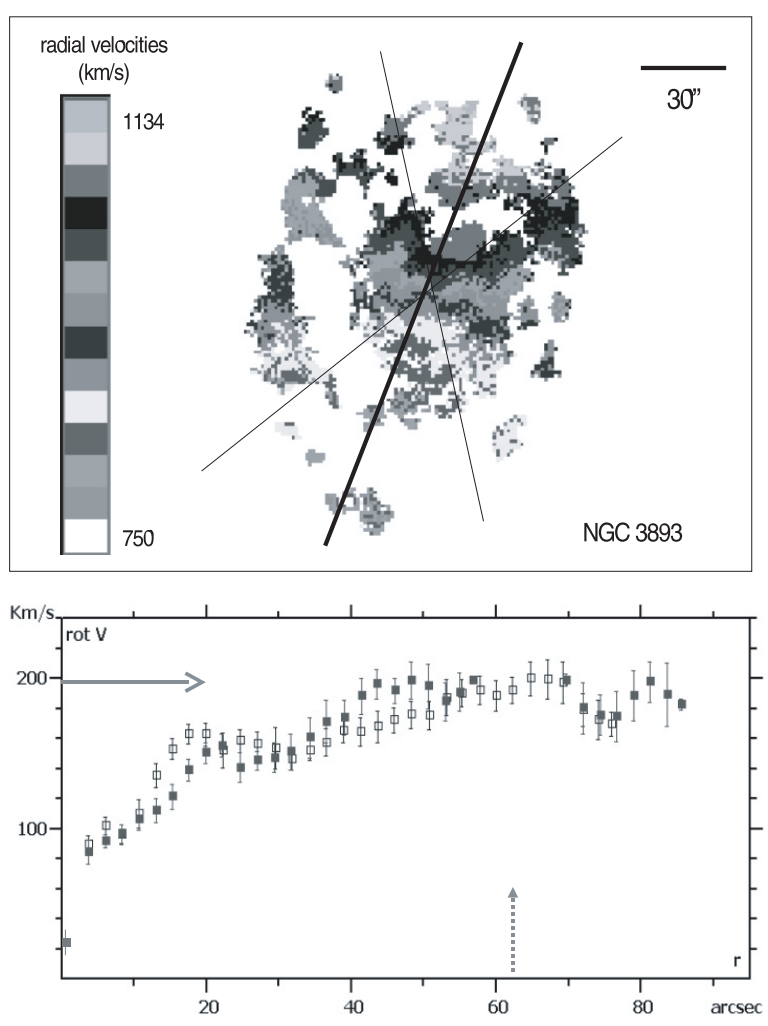

Fig. 2. Top: velocity field of NGC 3893 in KPG 302. The solid line indicates the galaxy's position angle (PA) and the slash-dotted lines indicate the angular sectors from both sides of the major axis considered for the computation of the galaxy's the computation of the galaxy's rotation curve. Bottom: rotation curve (RC) of NGC 3893. Both sides of the curve have been superposed. Open squares correspond to the receding side of the galaxy. Filled squares correspond to the approaching side. RC was plotted considering an inclination value of $45^{\circ}$. Horizontal solid arrow indicates the maximal rotation velocity. Vertical dotted arrow indicates the radius associated with corotation according to Kranz et al. (2003).

\subsubsection{NGC 3893}

The RC of NGC 3893 was computed considering points on the velocity field within an angular sector of $32^{\circ}$ on each side of the galaxy's PA. The kinematic center used to compute the rotation curve matches the photometric center from the PUMA continuum image within $1^{\prime \prime}$. The most symmetrical, smooth, and less-scattered RC was derived using the following set of values: $\mathrm{PA}=(340 \pm 10)^{\circ}, i=(45 \pm 3)^{\circ}$ and $V_{\text {sys }}=(962 \pm 5) \mathrm{km} \mathrm{s}^{-1}$. These are presented in Table 1 along with values obtained in previous works. The RC superposing both the approaching and receding sides is shown in the lower panel in Fig. 2. Globally the RC of NGC 3893 is symmetric up to the last $\mathrm{H} \alpha$ emission point at $85^{\prime \prime}$ $(7.55 \mathrm{kpc})$. Both sides of the curve display oscillations of about $10 \mathrm{~km} \mathrm{~s}^{-1}$. The maximum rotation velocity is $(197 \pm 10) \mathrm{km} \mathrm{s}^{-1}$ and is reached at $69^{\prime \prime}(6.1 \mathrm{kpc})$. Our RC is very similar to the one derived by Garrido et al. (2002) using FP observations. To compare with the results derived by Kranz et al. (2003), a long slit was simulated by considering radial velocities on the velocity field within a sector of $1.5^{\circ}$ on each side of the slit position angle. The values of the kinematic parameters were equal to those used by these authors. These "long-slit" RCs show the same increasing behavior and oscillations as those found by Kranz et al. (2003).
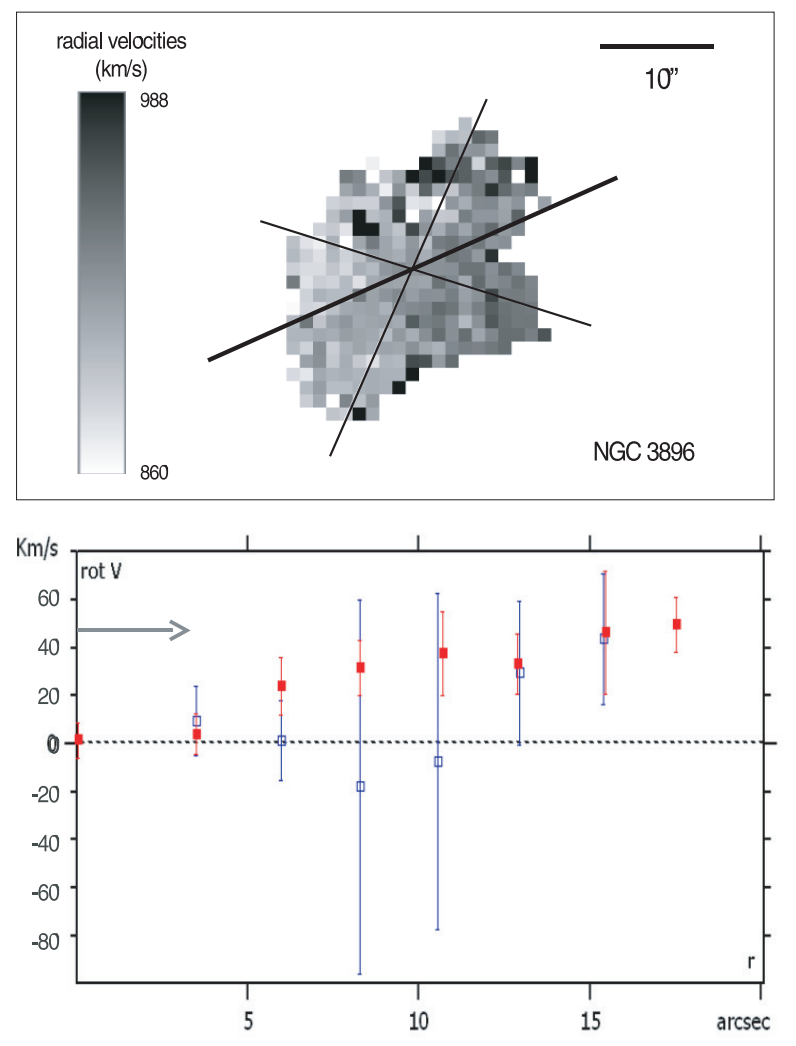

Fig. 3. Top: velocity field of NGC 3896 in KPG302. The solid line indicates the galaxy's position angle (PA) and slash-dotted lines indicate the angular sectors from both sides of the major axis considered for the computation of the galaxy's RC. Bottom: RC of NGC 3893. Both sides of the curve have been superposed. Open squares correspond to the receding side of the galaxy. Filled squares correspond to the approaching side. RC was plotted considering an inclination value of $49^{\circ}$. The solid arrow indicates maximal rotation velocity.

\subsubsection{NGC 3896}

Though the velocity field of this galaxy is very perturbed, we were still able to derive an RC reflecting the circular motions of the southwestern side of the galaxy (approaching side). The following set of values were used: $\mathrm{PA}=(294 \pm 5)^{\circ}, i=(49 \pm 3)^{\circ}$, and $V_{\text {sys }}=(920.50 \pm 5) \mathrm{km} \mathrm{s}^{-1}$, considering an angular sector of $42^{\circ}$ on each side of the galaxy's PA. These values, along with values from previous works, are presented in Table 1 . The lower panel in Fig. 3 shows the RC derived for this galaxy superposing both the approaching and receding sides. Globally the curve increases up to the last emission point at $17.5^{\prime \prime}(1.5 \mathrm{kpc})$ where the velocity equals $48 \mathrm{~km} \mathrm{~s}^{-1}$. Rather low velocity values appear near the center of the galaxy, within $4.2^{\prime \prime}(0.4 \mathrm{kpc})$.

\subsection{Non-circular motions}

Two-dimensional kinematic fields of disk galaxies portray the motion of the gas over the whole galaxy enabling us to match these motions with different morphological structures. One can determine to what extent the gas is following circular motion around the center of the galaxy and to what extent there are large contributions from non-circular velocities (radial, azimuthal, and vertical ) due to the presence of these structures or to external perturbations. For NGC 3893, we analyzed the influence of morphological features on the kinematics of the gas. This was done by comparing the monochromatic image with each side of the 


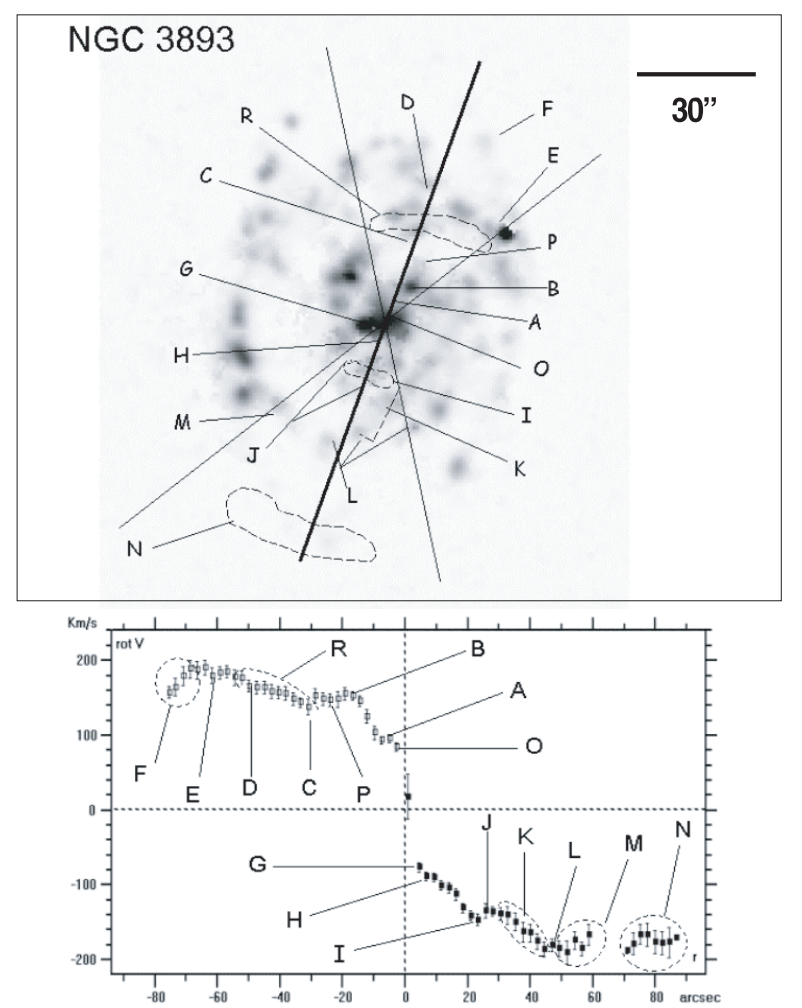

Fig. 4. Top: Monochromatic image of NGC 3893. Letters indicate features associated to points on the RC (shown in the bottom panel) in order to differentiate the contribution of circular from non-circular motions. Solid line indicates the galaxy's position angle (PA) and the thin lines indicate the angular sector from both sides of the major axis considered for the computation of the galaxy's RC.

RC (Fig. 4). Through this comparison we were able to differentiate points in the RC associated to circular motions of the gas associated with the global mass distribution of the galaxy - from non-circular motions - associated to the response of the gas to local morphological features.

\section{Dynamical analysis}

\subsection{Mass estimates through rotation curves}

The $\mathrm{H} \alpha$ kinematic information from our FP observations of NGC 3893 was complemented with HI synthesis observations by Verheijen \& Sancisi (2001). To match both curves, we considered the averaged optical rotation curve that was derived using the same kinematic parameters as those used for the HI RC (these values do not differ much from the values we used for the optical RC - see Table 1). The superposed curves are shown in Fig. 5. Both curves superpose smoothly except for the external parts of the optical $\mathrm{RC}\left(R \geq 60^{\prime \prime}\right)$, which appear to be more perturbed. The HI RC also decreases for $R \geq 175^{\prime \prime}$ and up to the last HI emission point at $R=245^{\prime \prime}(21.76 \mathrm{kpc})$. Verheijen \& Sancisi (2001) mention that the determination of the HI rotation curve beyond $120^{\prime \prime}$ is uncertain because of the tidal interaction with NGC 3896. We set this radius as our limit for the computation of the mass through the RC of NGC 3893.

Using this composite rotation curve, a range of possible masses was computed using the method proposed by Lequeux (1983) according to which the total mass of a galaxy within a radius $R$ lies between 0.6 (in the case of a disk-like mass distribution) and $1.0 \times\left(R V^{2}(R) / G\right)$ (in the case of a spheroidal mass

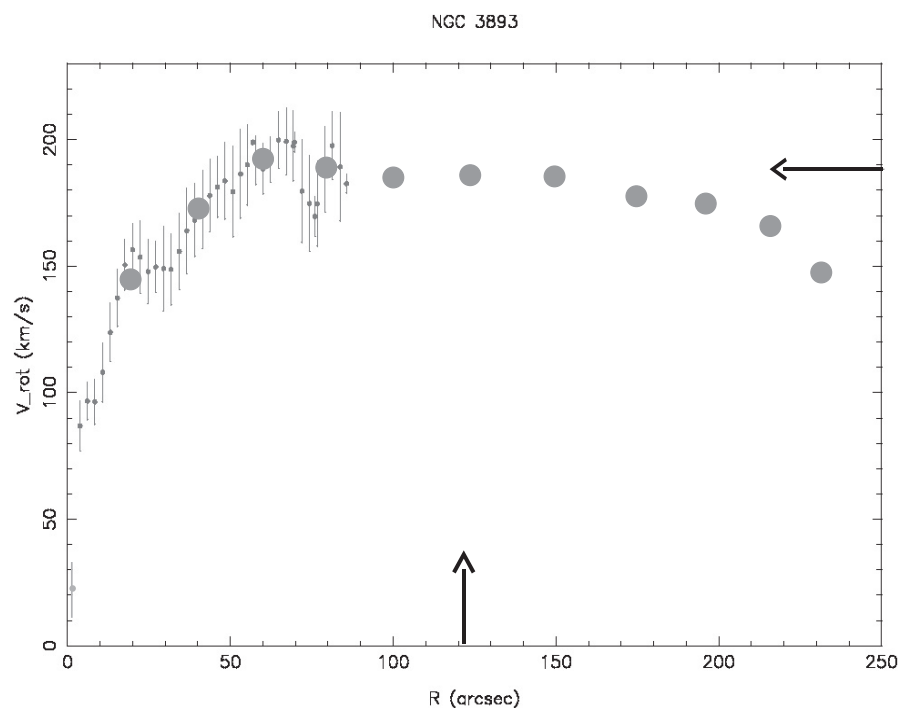

Fig. 5. Multi-wavelength curve of NGC 3893. Small dots in the inner parts of the curve correspond to optical Fabry-Perot $\mathrm{H} \alpha$ observations. Larger dots in the outer parts correspond to the HI curve derived by Verheijen \& Sancisi (2001). The horizontal arrow indicates the point with maximum rotation velocity. The vertical arrow shows the radius considered for mass estimation using the method by Lequeux (1983).

distribution). As a first estimate, we considered the maximum rotation velocity $\left(190 \mathrm{~km} \mathrm{~s}^{-1}\right)$ given by both the optical and radio RCs to estimate the mass within $R=120^{\prime \prime}=10.8 \mathrm{kpc}=$ $0.99 D_{25} / 2$. The range of masses within this radius is given by 0.50 to $0.84 \times 10^{11} M_{\odot}$. To derive the mass of NGC 3896 , we considered the last emission point on the approaching side of the curve, which shows the maximum amplitude of that curve. This value equals $48 \mathrm{~km} \mathrm{~s}^{-1}$ at $R=17.5^{\prime \prime}=1.5 \mathrm{kpc}=0.4 D_{25} / 2$. The masses within this radius range from 4.78 to $7.97 \times 10^{8} M_{\odot}$.

The mass ratio of the galaxies was computed from the NIR luminosities, calculated from the $K_{\mathrm{s}}$-magnitudes taken from 2MASS (Skrutskie et al. 2006). These luminosities for NGC 3893/96 lead to a mass ratio of 0.031 . On the other hand, the mass ratio derived from the $\mathrm{RC}$ of each galaxy within $0.4 D_{25} / 2$ falls around 0.0255 . These values are fairly similar, particularly if considering the uncertainty of the mass-to-light ratio. This strengthens the reliability of the mass estimates based on the rotation curves.

\subsection{Mass distribution}

We used the mass model from Blais-Ouellette et al. (2001) to study the mass distribution in NGC 3893. This model uses both the light distribution of the galaxy and a theoretical dark-halo profile to compute an RC that best-fits the observed one. The mass-to-light ratio of the disk $\left.(M / L)_{\text {disk }}\right)$, as well as the properties of the dark matter, characteristic density $\left(\rho_{0}\right)$, and radius $\left(R_{0}\right)$, are free parameters. We used a DM halo described by a pseudo-isothermal sphere (Begeman 1987) and a Navarro, Frenk \& White (NFW) profile (Navarro et al. 1996) to fit the multiwavelength RC of NGC 3893. Optical photometry in the $I$ band was taken from Hernández-Toledo \& Puerari (2001) and the HI superficial distribution from Verheijen \& Sancisi (2001). Making use of the possibility of disentangling circular from non-circular velocities in the optical RC, our multi-wavelength $\mathrm{RC}$ was "cleaned" from points associated to non-circular motions (see Sect. 4.4) and to the warp in the outer parts of the HI disk. We removed points in the optical part of the observed RC between 


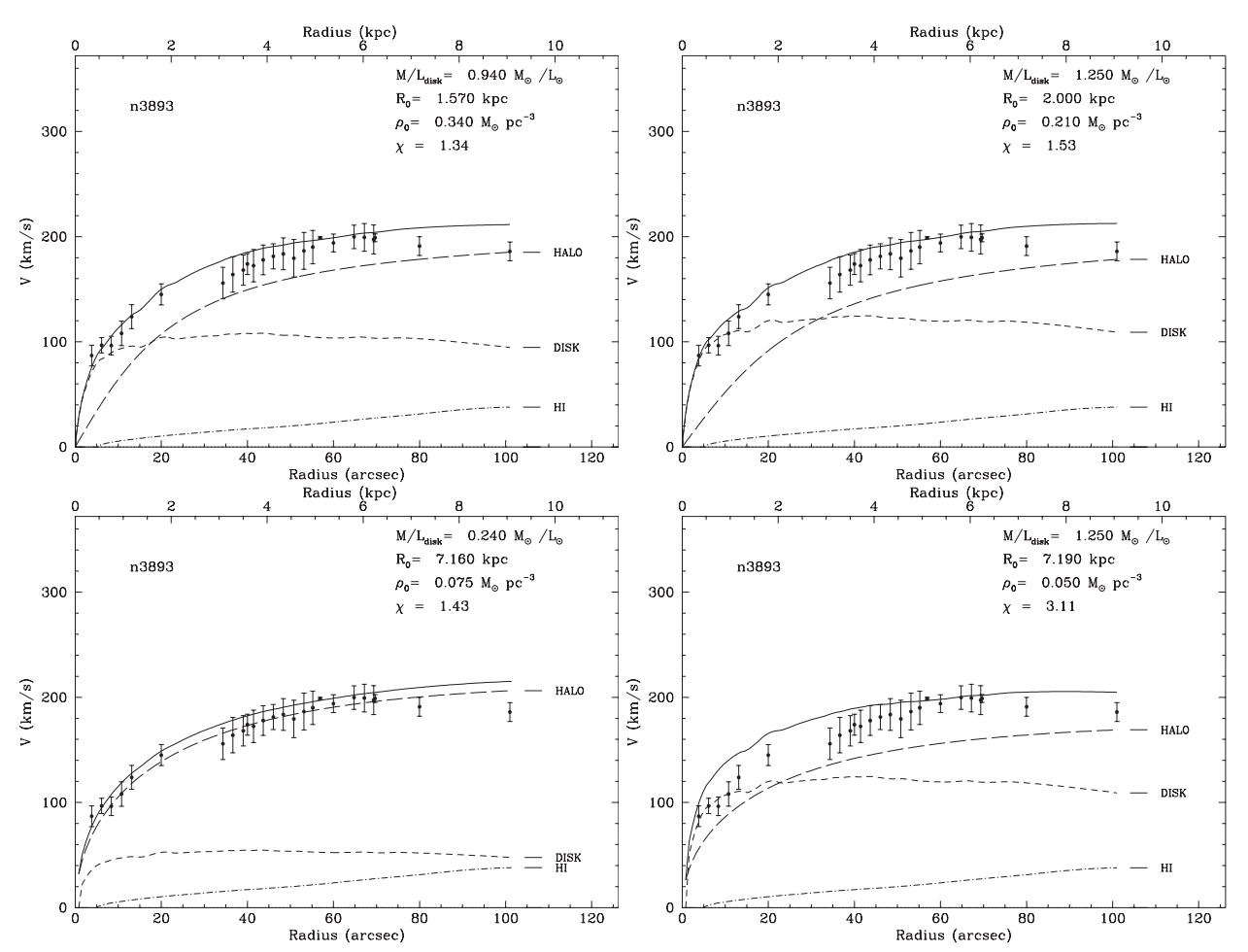

Fig. 6. Best mass model fits for the multi-wavelength rotation curve of NGC 3893 once the points associated with non-circular motions have been removed. Top left: pseudo-isothermal halo and non-maximal disk. Top right: pseudo-isothermal halo and maximal disk. Bottom left: NFW halo and non-maximal disk. Bottom right: NFW halo and maximal disk. The long-dashed curve represents the dark-matter halo contribution and the short-dashed curve represents the stellar disk contribution. The parameters displayed stand for the mass-to-light ratio of the stellar disk $\left(M / L_{\mathrm{disk}}\right)$, the characteristic radius of the dark-matter halo and density $\left(R_{0}\right.$ and $\rho_{0}$, respectively) and the minimized $\chi^{2}$ in the three-dimensional parameter space. Mass-model taken from Blais-Ouellette et al. (2001).

$15^{\prime \prime}-20^{\prime \prime}, 22^{\prime \prime}-32^{\prime \prime}$, and at $R>75^{\prime \prime}$ (see Fig. 5). Only points from the $\mathrm{HI}$ rotation curve were taken into account after $75^{\prime \prime}$ and up to $120^{\prime \prime}$ considering the fact that the $\mathrm{RC}$ in $\mathrm{HI}$ is uncertain beyond this radius.

Figure 6 shows different fits for this multi-wavelength RC: a pseudo-isothermal DM halo with non-maximal disk (top left), a pseudo-isothermal DM halo with a maximal disk (top right), a NFW halo with non-maximal disk (bottom left), and an NFW halo with a maximal disk (bottom left). Table 2 displays the mass-model parameters used in each case. We used the definition of Sackett (1997) for the "maximal disk" that is taken to be a galactic disk such that $85 \% \pm 10 \%$ of the total rotational support of a galaxy at a radius $2.2 \times$ scale radius is contributed by the stellar-disk mass component. For NGC 3893, this radius corresponds to $1.80 \mathrm{kpc}\left(\mathrm{Kranz}\right.$ et al. 2003). The best fit $\left(\chi^{2}=1.34\right)$ is obtained using a pseudo-isothermal halo with a non-maximal disk leading to $(M / L)_{\text {disk }}=0.94$ in the $I$ band, yet it misses the last two points of the RC. The fit with an NFW halo and a non-maximal disk gives $(M / L)_{\text {disk }}=0.24\left(\chi^{2}=1.43\right)$, also missing the two outermost points of the RC. Both a pseudoisothermal and NFW halo with a maximal disk give higher values of $(M / L)_{\text {disk }}(1.25$ in both cases $)$ and also higher $\chi^{2}$ values (1.53 and 3.11, respectively). They also miss both outer and inner points on the RC.

To evaluate the effect of non-circular motions on the fits, we fitted the above mass models by including the points associated to non-circular motions to the multi-wavelength $\mathrm{RC}$. The fits can be seen in Fig. 7. The values for the mass-model parameters are shown in Table 2 . These fits are less precise. In all cases, the mass-to-luminosity ratio using the maximal disk assumption is higher than the one obtained by Kranz et al. (2003)
$\left((M / L)_{\text {disk }}=0.56-\right.$ in both $K$ and $I$ bands $)$. We must take into account that Kranz et al. (2003) only fit the optical part of the $\mathrm{RC}$ derived with long-slit spectroscopy. For the sake of comparison, we fitted our $\mathrm{H} \alpha \mathrm{RC}$ (with and without points associated to non-circular motions) using the value for $(M / L)_{\text {disk }}$ derived by these authors. Results are shown in Fig. 8 and Table 2. Fits are very good for both the pseudo-isothermal and the NFW halo; nevertheless, $(M / L)_{\text {disk }}=0.56$ does not render the disk maximal. Finally we also fitted these mass models to the HI RC without points at $R>120^{\prime \prime}$ where tidal effects might affect the correct determination of the RC. Figure 9 and Table 2 show the resulting parameters of the fits. The fit using the NFW halo misses the innermost point of the curve and is not very accurate $\left(\chi^{2}=3.17\right)$. The fit using the pseudo-isothermal halo misses the middle point of the curve, yet $\chi^{2}=1.32-$ which is one of the lowest values found for all fits presented. Nevertheless it should be noticed that for both halos, the $(M / L)_{\text {disk }}>1.7$, which is higher than the value found with the multi-wavelength $\mathrm{RC}$. This highlights the importance of the multi-wavelength approach for the mass model.

\section{Discussion}

That no model fits the last point in the multi-wavelength $\mathrm{RC}$ of NGC 3893 "cleaned" from the effects of non-circular motions can be explained either by a truncated halo for this galaxy or by the existence of a common halo for both galaxies. Since the derived mass of the NGC 3896 is low, then it most probably resides inside the halo of NGC 3893, and both galaxies share a single halo. Nevertheless this halo would have a different distribution than in an isolated galaxy. When considering an isothermal 
Table 2. Mass models parameters for NGC 3893 from best fits of the multi-wavelength rotation curve, considering only points associated to circular motions.

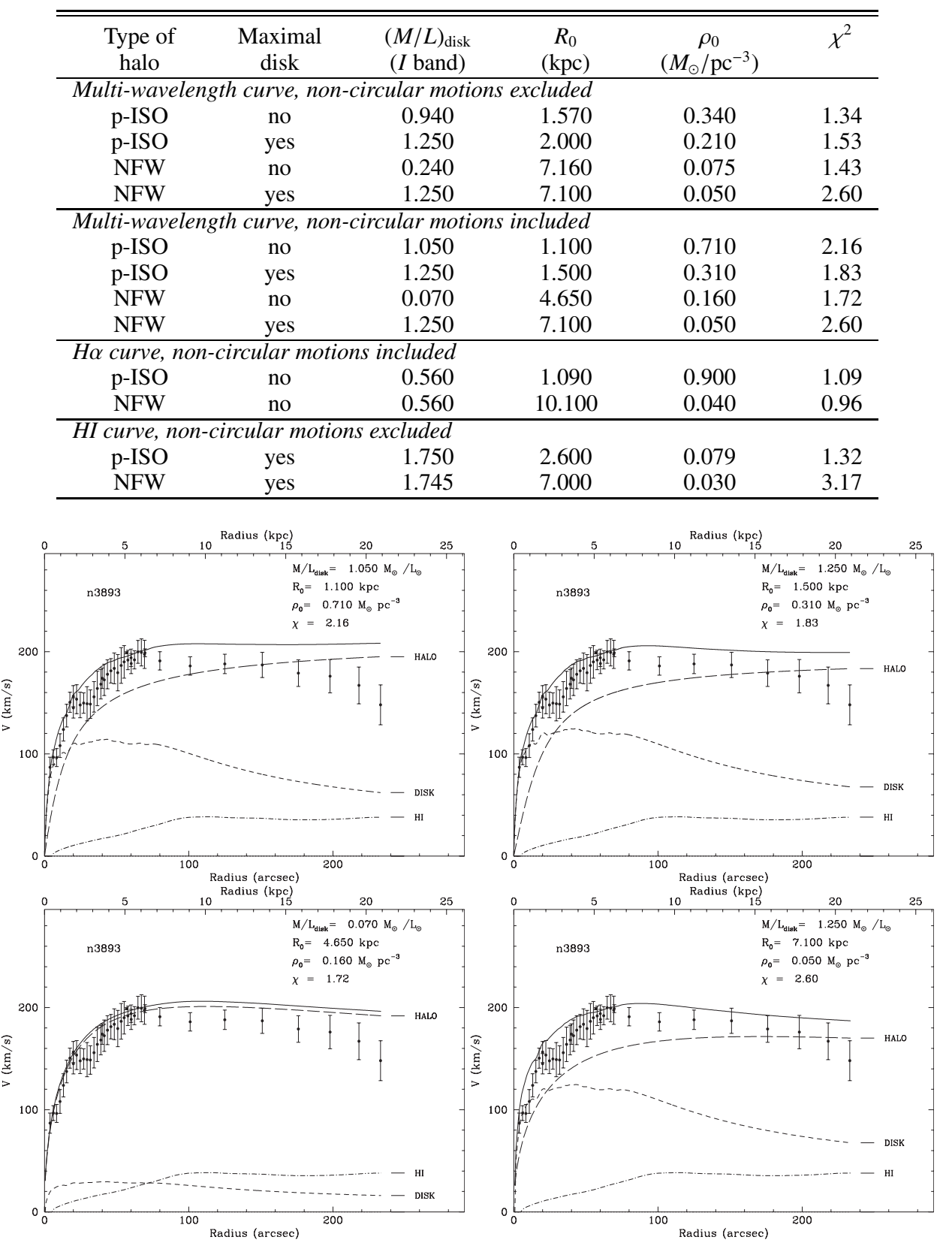

Fig. 7. Best mass-model fit for the multi-wavelength rotation curve of NGC 3893 considering all observed points - including those associated with non-circular motions. Top left: pseudo-isothermal halo and non-maximal disk. Top right: pseudo-isothermal halo and maximal disk. Bottom left: NFW halo and non-maximal disk. Bottom right: NFW halo and maximal disk.

halo and the HI curve, the $(M / L)_{\text {disk }}$ that fits this curve best is much higher than the $M / L$ found for the multi-wavelength curve. In general, the information on the inner parts of the galaxy given by the optical observations imposes an $(M / L)_{\text {disk }} \leq 1$, which would imply the presence of a disk with a large population of young stars -which is not the case given the $B-V$ value derived by Hernández-Toledo \& Puerari (2001). This supports the idea that the structure of the DM halo of this pair differs from that of a single-disk galaxy. As shown by Laurikainen \& Salo (2001), M 51-type pairs companions tend to have extremely large bulge sizes relative to their disk scale-lengths. Consequently, the bulge-to-disk luminosity ratios for the companions were also generally higher than known for any of the
Hubble types of normal galaxy. This is the case for NGC 3896 , whose RC displays almost solid body rotation up to the last emission point.

\section{Conclusions}

We have presented the kinematic and dynamical analysis of the M 51-type galaxy pair, KPG 302 (NGC 3893/96). NGC 3893 is a grand-design spiral with a regular velocity field that displays no major distortions. The companion, NGC 3896, displays ongoing star formation that was probably triggered by the interaction with the main galaxy. This galaxy displays important noncircular motions in localized regions, especially on the side of 

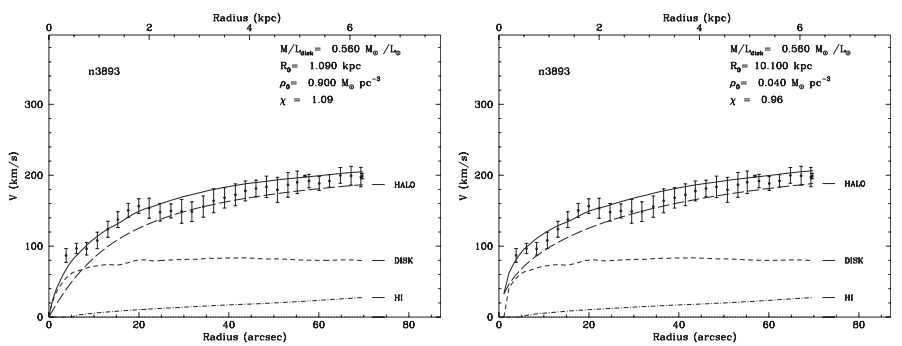

Fig. 8. Best mass-model fit for the $\mathrm{H} \alpha$ rotation curve of NGC 3893 considering all observed points -including those associated with noncircular motions and using the $(M €)_{\text {disk }}$ value by Kranz et al. (2003). Left: pseudo-isothermal halo. Right: NFW halo.
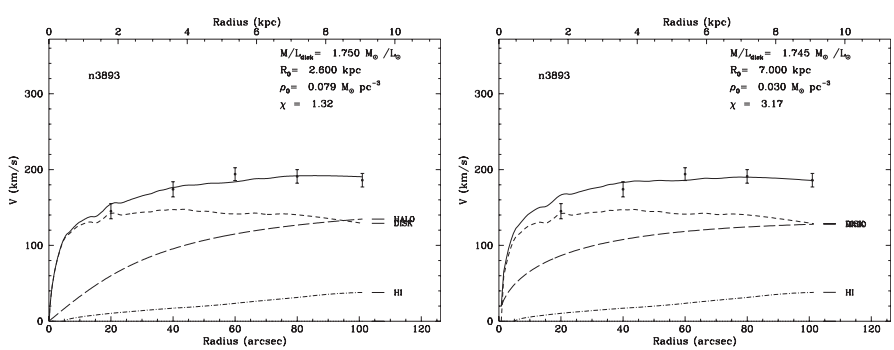

Fig. 9. Best mass-model fit for the HI rotation curve of NGC 3893 after removing points associated with non-circular motions and the warp of the outer parts of the disk. Left: pseudo-isothermal halo. Right: NFW halo.

the galaxy that is closer to the companion. The total mass of each galaxy was derived from the RC. The optical RC of NGC 3893 was matched with the existing HI curve to determine the distribution of the luminous and dark matter. This multi-wavelength rotation curve was analyzed in light of the $3 \mathrm{D}$ observations in order to differentiate the contribution of non-circular motions associated to particular features and the contribution of circular motions, which reflect the mass distribution of the galaxy. No "classical" DM halo fits the observed rotation curve, which could imply a different mass distribution for the DM halo of M 51-type binary galaxies.
Acknowledgements. We wish to thank the staff of the Observatorio Astronómico Naconal (OAN-SPM) for their support during PUMA data acquisition. We also thank C. Carignan for letting us use his mass model. I.F.C. acknowledges the financial support of FAPESP and CONACYT grants No. 03/01625-2 and No. 121551 - respectively. M.R. acknowledges financial support from grants 46054-F from CONACYT and IN100606 from DGAPA-UNAM. H.S. and E.L. acknowledge the support from the Academy of Finland. We acknowledge the use of the HyperLeda database (http://leda. univ-lyon 1.fr), the NASA/IPAC Extragalactic Database (NED), and the Two Micron All Sky Survey.

\section{References}

Begeman, K. G. 1987, Ph.D. Thesis, Groningen University

Blais-Ouellette, S., Amram, P., \& Carignan, C. 2001, AJ, 121, 1952

Fuentes-Carrera, I., Rosado, M., Amram, P., et al. 2004, A\&A, 415, 451

Garrido, O., Marcelin, M., Amram, P., \& Boulesteix, J. 2002, A\&A, 387, 812

Garrido, O., Marcelin, M., Amram, P., Balkowski, C., \& Boulesteix, J. 2005, MNRAS, 362, 127

Hernández-Toledo, H., \& Puerari, I. 2001, A\&A, 379, 54

James, P. A., Shane, N. S., Beckman, J. E., et al. 2004, A\&A, 414, 23

Karachentsev, I. D. 1972, Catalogue of isolated pairs of galaxies in the northern hemisphere, Soobshch. Spets. Astrofiz. Obs, 7, 1

Knebe, A., Gill, S. P. D., \& Gibson, B. K. 2004, PASA, 21, 216

Kranz, T., Slyz, A., \& Rix, H.-W. 2003, ApJ, 586, 143

Laurikainen, E., \& Salo, H. 2001, MNRAS, 324, 685

Laurikainen, E., Salo, H., \& Aparicio, A. 1998, A\&A, 129, 517

Le Coarer, E., Rosado, M., Georgelin, Y., Viale, A., \& Goldes, G. 1993, A\&A, 280,365

Lequeux, J. 1983, A\&A, 125, 394

Navarro, J. F., Frenk, C. S., \& White, S. D. M. 1996, ApJ, 462, 563

Pasha, I. I., \& Smirnov, M. A. 1982, Ap\&SS, 86, 215

Rosado, M., Langarica, R., Bernal, A., et al. 1995, RevMexAA (Serie de Conferencias), 03, 263

Sackett, P. D. 1997, ApJ, 483, 103

Salo, H., Rautiainen, P., Buta, R., et al. 1999, AJ, 117, 792

Sandage, A., \& Bedke, J. 1994, The Carnegie Atlas of Galaxies. Volume II, Carnegie Institution of Washington with The Flintridge Foundation

Skrutsie, M. F., Cutri, R. M., Stiening, R., et al. 2006, AJ, 131, 1163

Sofue, Y., \& Rubin, V. 2001, ARA\&A, 39, 137

Struck, C. 2005 [arXiv: astro-ph/0511335]

Tully, R. B., Verheijen, M. A. W., Pierce, M. J., Huang, J.-S., \& Wainscoat, R. J. 1996, AJ, 112, 2471

de Vaucouleurs G., de Vaucouleurs A., Corwin H. G., et al. 1991, Third Reference Catalogue of Bright Galaxies (RC3) (New York: Springer-Verlag) Verheijen, M. A. W. 2001, ApJ, 563, 694

Verheijen, M. A. W., \& Sancisi, R. 2001, A\&A, 370, 765 